\title{
A Status Data Transmitting System for Vessel Monitoring
}

\author{
The Anh Nguyen Dinh ${ }^{1}$, Huy Le Xuan ${ }^{2}$, Tuan Anh Vu ${ }^{3}$, and Duong Bach Gia \\ ${ }^{1,2}$ Vietnam National Space Center, Vietnam Academy of Science and Technology, Vietnam \\ ${ }^{3,4} \mathrm{VNU}$ University of Engineering and Technology, Vietnam
}

\begin{tabular}{l}
\hline Article Info \\
\hline Article history: \\
Received September 7, 2017 \\
Revised: Jan 3, 2018 \\
Accepted: Jan 28, 2018 \\
\hline
\end{tabular}

Keyword:

Marine communications

Wideband synthesizer

Mixer

VCO-PLL

Horn antenna

\begin{abstract}
This paper presents a status data transmitting system suitable for vessel monitoring. The system consists of four main parts, which are a frequency synthesizer, a horn antenna, a status data module and a power amplifier. The proposed frequency synthesizer can flexibly change the frequency in a wide range (from $600 \mathrm{MHz}$ to $4.2 \mathrm{GHz}$ ) and the output power (from $-6 \mathrm{dBm}$ to $-3 \mathrm{dBm}$ ). By using the phase-locked loop, the synthesizer's stability over temperature and tolerance are comparable to temperature compensated crystal oscillators (TCXO) that is about $+/-3 \mathrm{ppm}$. Moreover, phase noise performance of the synthesizer is less than $-90 \mathrm{dBc} / \mathrm{Hz}$ at $1 \mathrm{KHz}$ and $-100 \mathrm{dBc} / \mathrm{Hz}$ at $100 \mathrm{KHz}$. The impedance bandwidth of the horn antenna can be controlled by using the beveling technique. The status data module packs information of the identification, longitude, latitude, and state of the vessel into data frames. FSK/MSK/GMSK schemes were used to modulate the data. The power amplifier provides $130 \mathrm{~W}$ output power at S-band. The fundamental characteristics of the implemented transmitter have been measured and verified.
\end{abstract}

Copyright (C) 2018 Institute of Advanced Engineering and Science. All rights reserved.

\section{Corresponding Author:}

Name: The Anh Nguyen Dinh

Affiliation: Vietnam National Space Center (VNSC), Vietnam Academy of Science and Technology

Address: VNSC Building (A6), 18 Hoang Quoc Viet, Hanoi, Vietnam

Phone: +840902061090

Email: ndtanh@vnsc.org.vn

\section{INTRODUCTION}

Recently, maritime security has become a major concern of all coastal countries, and the fundamental requirement is maritime domain awareness via identification, tracking, and monitoring of vessels within their waters [1]. Ref. [1] briefly presented the recent technologies and systems for different types of vessels. These systems have been designed and provided with a regulatory framework for other reasons such as sustainable fishery, search and rescue services, environmental protection, navigational safety, etc. However, the main problem is the distance of communications. The conventional methods such as GSM, UHF, VHF are limited by the communication distance, which is less than $100 \mathrm{~km}$ [2], [3]. Recently, satellite technology has extremely developed and become the best method to solve the above problem [4].

In this paper, we are going to present the designs and measurement results of a status data transmitting system as illustrated in Fig. 1. The system is able to flexibly change the transmitting parameters such as frequency, power level, mode of modulation, and state of a vehicle. In addition, the frequency stability over temperature and frequency tolerance of the system are $+/-2.5 \mathrm{ppm}$ and $+/-3 \mathrm{ppm}$, respectively. The paper is organized as follow. Section 2 introduces the architectures of the proposed status data transmitting system including detailed descriptions of each building block. The results are presented in section 3 and conclusions are given in the last section.

\section{DESIGN OF THE STATUS DATA TRANSMITTING SYSTEM}

The information is packed into data frames including the identification, longitude, latitude, and state of a vessel $(<\mathrm{GPS}>,<\mathrm{ID}>,<\mathrm{LAT}>,<\mathrm{LONG}>,<\mathrm{SOS}>$ ). The proposed status module uses ADF7021 transceiver from Analog Device to process the data. The ADF7021 transceiver is a high performance, low power, highly integrated 2FSK, 3FSK, 4FSK, MSK, GMSK transceiver. It is designed to operate in the narrow-band, license-free 


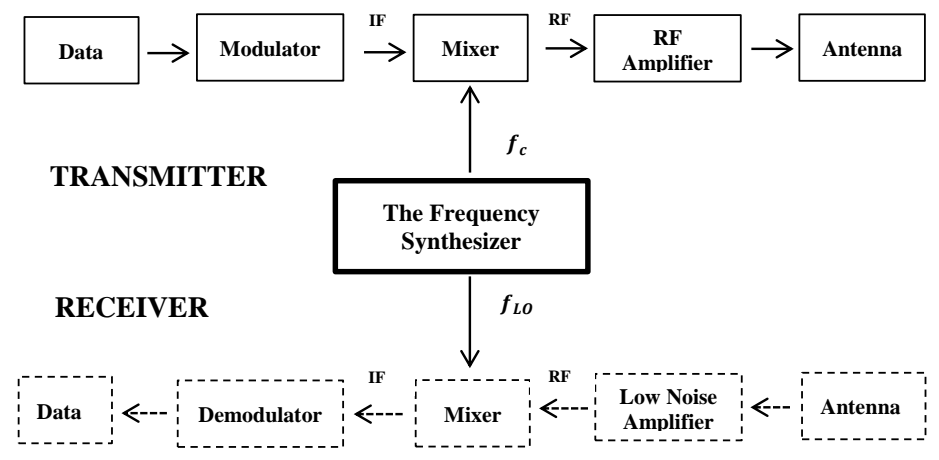

Figure 1. The proposed status data transmitting system

ISM bands, and in the licensed bands with frequency ranges from $80 \mathrm{MHz}$ to $650 \mathrm{MHz}$ and $862 \mathrm{MHz}$ to $950 \mathrm{MHz}$. This device has both Gaussian and raised cosine transmitting data filtering options to improve spectral efficiency for narrow-band applications. A LC bandpass filter was designed to have $50 \mathrm{~dB}$ out-of-band attenuation for the suppression of harmonics as shown in Fig. 2. In this design, the status data module is integrated with a LTC5510 mixer from Linear Technology. The LTC5510 is a high linearity mixer optimized for applications requiring very wide input bandwidth, low distortion, and low LO leakage. The mixer includes a double-balanced active mixer with an input buffer and a high speed LO amplifier. The input is optimized to use with 1:1 transmission line baluns, allowing very wideband impedance matching. Measurement results of the status module were presented in [5].

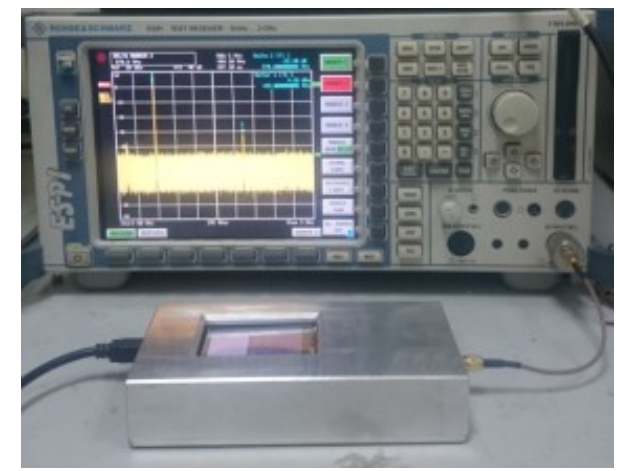

Figure 2. The proposed status module

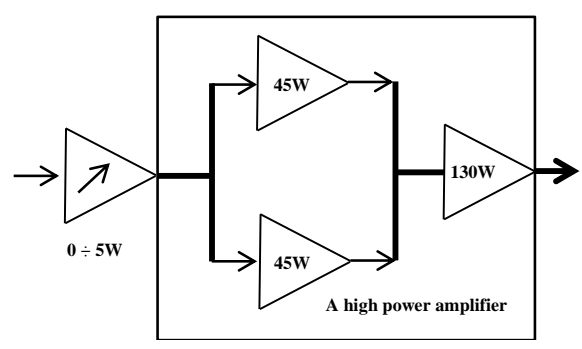

Figure 3. The structure of the Power Amplifier

The $80 \mathrm{~W}$ and $130 \mathrm{~W}$ power amplifiers were presented in [6] and [7], respectively. The simulation results were obtained using a well-known professional design software for microwave engineering, Advanced Design System 2009. The performance of the power amplifier modules were verified experimentally using a vector network 
analyzer. As illustrated in Fig. 3, a high power amplifier based on two mentioned amplifier modules was fabricated. Measurement results show that the power amplifier obtains a maximum output power of $130 \mathrm{~W}$ at S-band.

\subsection{The Frequency Synthesizer}

In general, high speed frequency synthesizers are divided into two types including digital direct synthesizer (DDS) and phase-looked loop (PLL) frequency synthesizer. The DDS has high conversion rate and high frequency resolution. However, the drawback of DDS is that it is not flexible in changing the step of the transmitting frequency. As a result, DDS is not suitable for maritime applications. In comparison with DDS, PLL synthesizer's advantages are high spurious suppression, relatively simple frequency control, and achievable broadband frequency source [8] [9]. The main part of PLL frequency synthesizer is a phase-looked loop. The phase-looked loop is a phase feedback system. It mainly consists of a VCO, loop filter (LF), and a phase comparator (PC). Through the phase comparator, the output frequency of VCO can accurately track the change of the input signal [10] [11]. In this design, the PLL frequency synthesizer was employed.

The frequency synthesizer based on the ADF4350's integrated PLL and the STM32F103C8 microcontroller are designed and implemented [12],[13]. The block diagram of the frequency synthesizer is illustrated in Fig. 4.

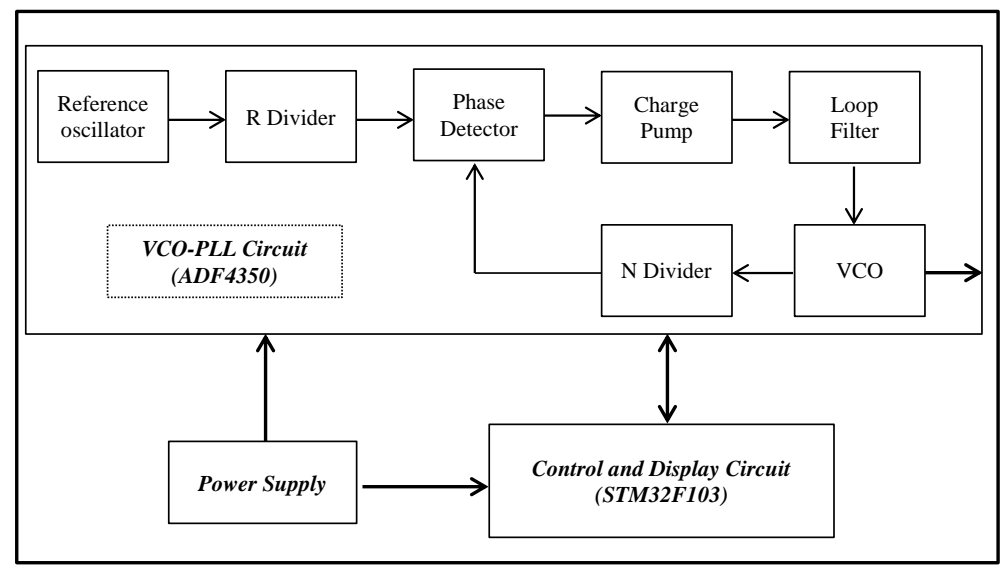

Figure 4. The block diagram of the frequency synthesizer

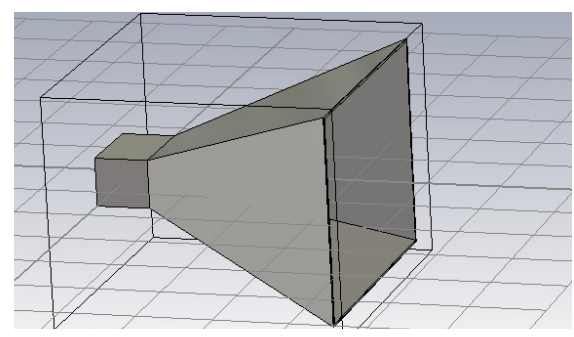

Figure 5. The horn antenna

\subsection{The Horn Antenna}

Horn antennas are one kind of aperture antennas as shown in Fig. 5, which provides the moderately high gain compared to other antennas. They are now commonly used for many applications including microwave communications, feeds for reflector antennas and radar elements. Horn antennas provide high gain, low VSWR, relatively wide bandwidth and high power handling properties. Recently, many techniques have been proposed for wideband planar monopole antennas such as notches, beveling, lumped circuit, and various shapes. In this design, we have applied the beveling technique to control the impedance bandwidth and increase the bandwidth with good control of the edge frequency. 


\section{MEASUREMENT RESULTS}

\subsection{The Frequency Synthesizer}

In order to validate the performance of the frequency synthesizer, a prototype was fabricated as shown in Fig. 6. The control and display circuit was fabricated on two-layer FR4 material with the dimension of $7 \mathrm{~cm} \times 14$ $\mathrm{cm}$. The PLL with integrated VCO was fabricated on multi-layers board technology with a small dimension of 5.5 $\mathrm{cm} \times 6 \mathrm{~cm}$ while the output impedance is matched over the wide frequency band. In total, the designed frequency synthesizer has a dimension of $9 \mathrm{~cm} \times 16 \mathrm{~cm} \times 3.5 \mathrm{~cm}$. The proposed frequency synthesizer was measured using a

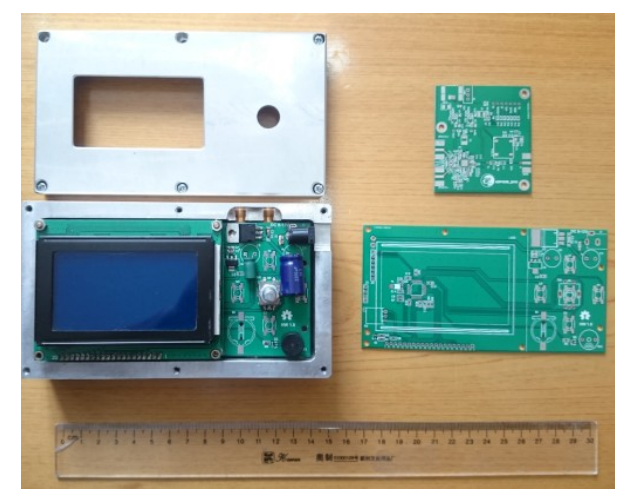

Figure 6. The fabricated frequency synthesizer

Rohde \& Schwarz spectrum analyzer. Fig. 7 shows the measured output power of the proposed synthesizer. As can be seen in the figure, the output power is from $-6 \mathrm{dBm}$ to $-3 \mathrm{dBm}$ over a wide frequency range. The operational frequency can be flexibly changed from $600 \mathrm{MHz}$ to $4.2 \mathrm{GHz}$. With the proposed design, the frequency stability over temperature and frequency tolerance are comparable to TCXO that are $+/-2.5 \mathrm{ppm}$ and $+/-3 \mathrm{ppm}$, respectively. Fig. 8 shows the phase noise performance which is less than $-90 \mathrm{dBc} / \mathrm{Hz}$ at $1 \mathrm{KHz}$ and $-100 \mathrm{dBc} / \mathrm{Hz}$ at $100 \mathrm{KHz}$. Table 1 summarizes the performance of the proposed frequency synthesizer and compares it to other published designs operating in a similar frequency range.

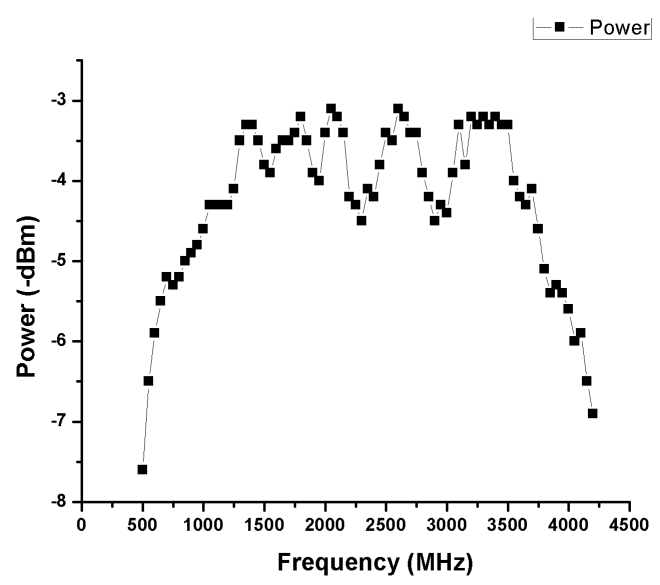

Figure 7. The measured output power of the proposed synthesizer

\subsection{The Horn Antenna}

All designs and simulations of the horn antenna were carried out using CST Microwave Studio. After optimizing, a prototype antenna was fabricated as shown in Fig. 9. It is then measured by using the Vector Network Analyzer (VNA) and NSI 2000 Near-field System. Fig. 10 illustrates the feed probes of the horn antenna. A monopole (a) is chosen for horn antennas. In this design, the beveling technique $(b, c)$ is used to improve the bandwidth of the antenna. The edge frequencies can be controlled by beveling all sides of the feed probe $(\mathrm{d} 1, \mathrm{~d} 2,11,12, \mathrm{~b} 1, \mathrm{~b} 2)$. Fig. 11 


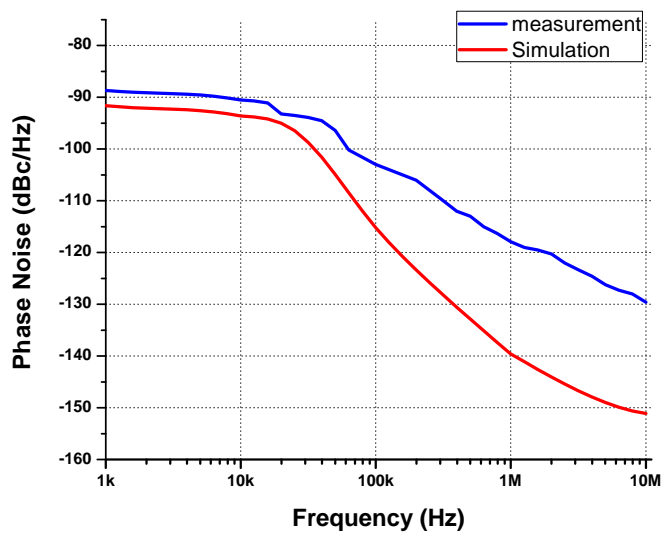

Figure 8 . The measured and simulated phase noise of the proposed sysnthesizer

Table 1. Comparison with the recent published works

\begin{tabular}{lcr}
\hline References & Frequency $(\mathrm{MHz})$ & Phase noise $(\mathrm{dBc} / \mathrm{Hz})$ \\
\hline Ref. [14] & $3385-3457$ & better than -80 at $20 \mathrm{KHz}$ \\
Ref. [15] & $800-4000$ & - \\
Ref. [16] & $137.5-4400$ & -90 at $100 \mathrm{KHz}$ \\
Ref. [17] & $210-4400$ & better than -80 at $1 \mathrm{KHz}$ \\
This work & $600-4200$ & -90 at $1 \mathrm{KHz},-100$ at $20 \mathrm{KHz},-110$ at $100 \mathrm{KHz}$ \\
\hline
\end{tabular}

shows the simulated input return loss of the horn antenna in three cases. In the first case, the feed probe is a monopole as illustrated in Fig. 10a. The bandwidth of the antenna is about $500 \mathrm{MHz}$. In the second case, when the feed probe is beveled as depicted in Fig. 10b, the bandwidth is extended from $500 \mathrm{MHz}$ to $1000 \mathrm{MHz}$. In the third case, the bandwidth is increased from $1000 \mathrm{MHz}$ to $1200 \mathrm{Mhz}$ when the feed probe as shown in Fig. 10c is used. The designed antenna can operate at both $\mathrm{L}$ band and $\mathrm{S}$ band. Fig. 12 plots the measured and simulated input return loss of the designed antenna. As can be seen, the measured result shows a good agreement with the simulated one. The gain of the antenna is shown in Fig. 13. It archives a maximal gain of $17.5 \mathrm{dBi}$ at $2.8 \mathrm{GHz}$. Fig. 14 and Fig. 15 show the radiation pattern and beamwidth of the designed antenna with very low sidelobes.

Table 2. Dimensions of the proposed antenna

\begin{tabular}{lc}
\hline Parameters & Value $(\mathrm{mm})$ \\
\hline $\mathrm{L}$ & 385 \\
$\mathrm{~W}$ & 290 \\
$\mathrm{H}$ & 398 \\
\hline
\end{tabular}

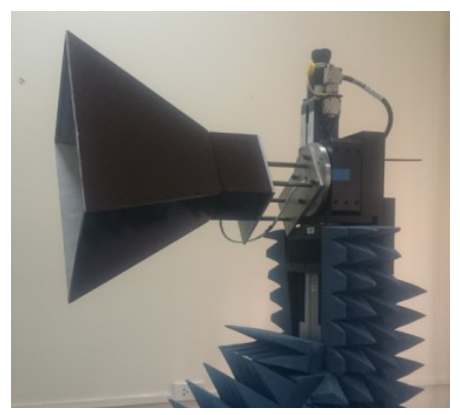

Figure 9. Measuring the antenna with NSI 2000 Near Field System 

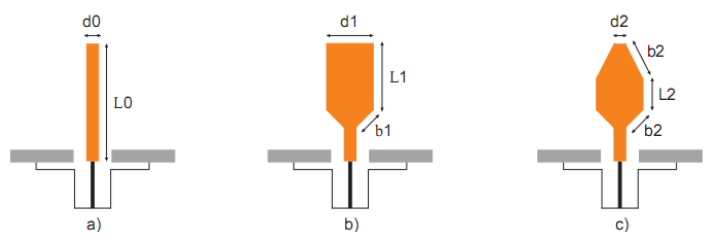

Figure 10. Feed probes of the horn antenna

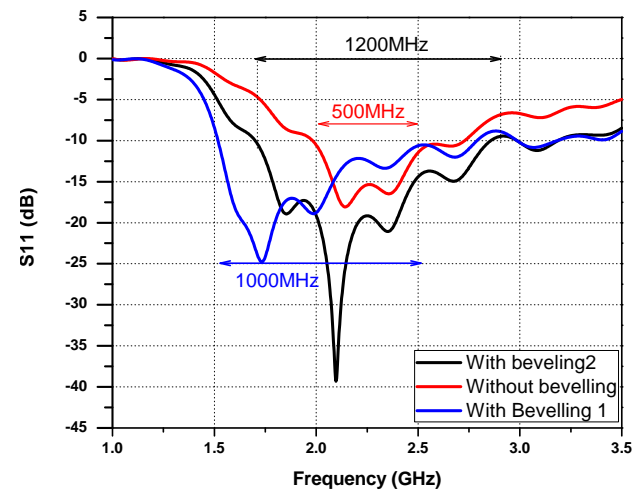

Figure 11. Bandwidth improvement by using bevelling technique

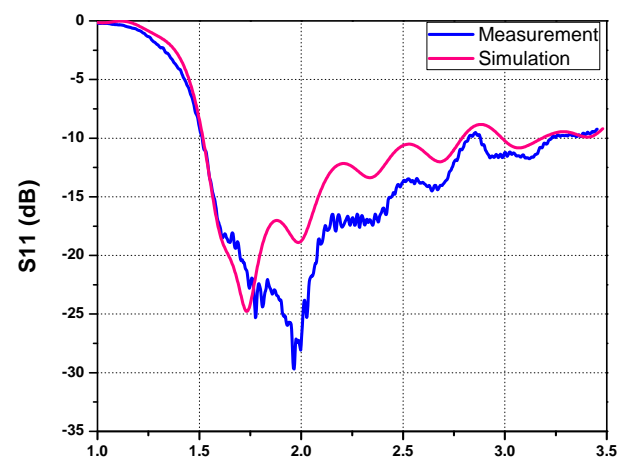

Figure 12. The measured and simulated input return loss of the designed horn antenna

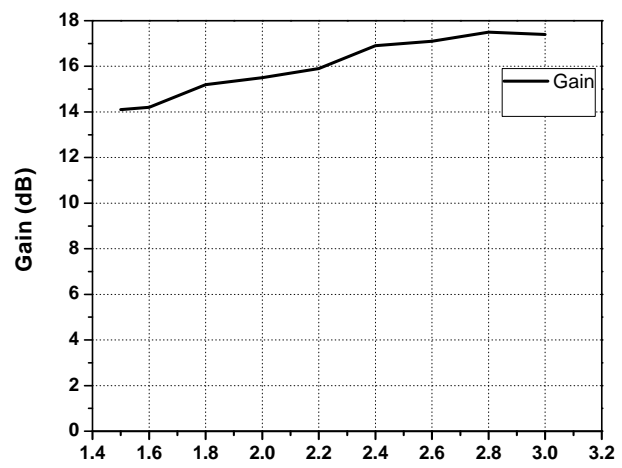

Figure 13. The measured gain of the designed horn antenna

\subsection{The Proposed System}

The information of vehicles is displayed on computers' screen by using our software. This helps the owners monitor the information and the state of the vessels as well as increase the safety on the sea. The proposed system was tested in a small area within a radius of $2 \mathrm{~km}$. The result is illustrated in Fig. 16, 17. Our software uses the 


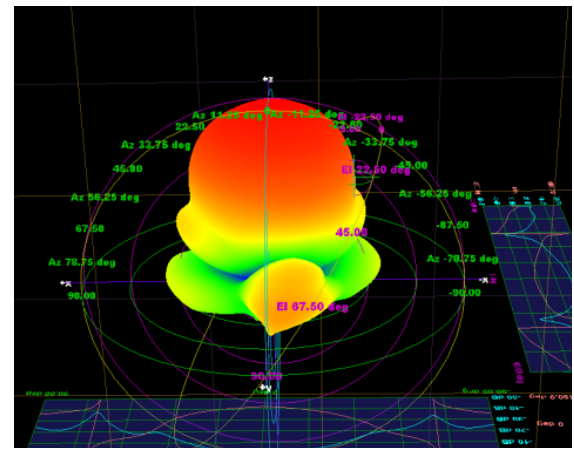

Figure 14. The measured radiation pattern of the designed horn antenna

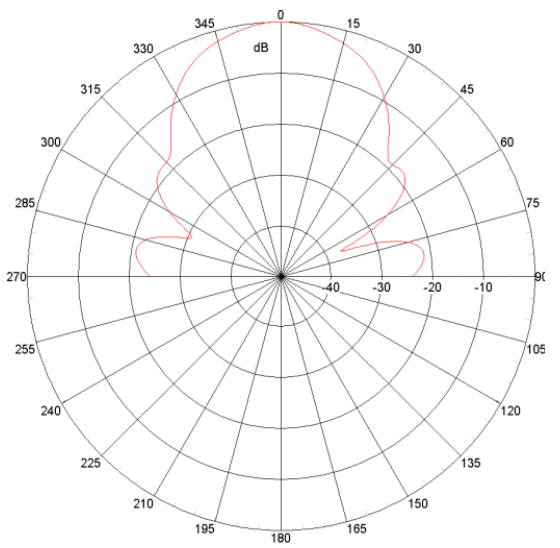

Figure 15. The measured beamwidth of the designed horn antenna

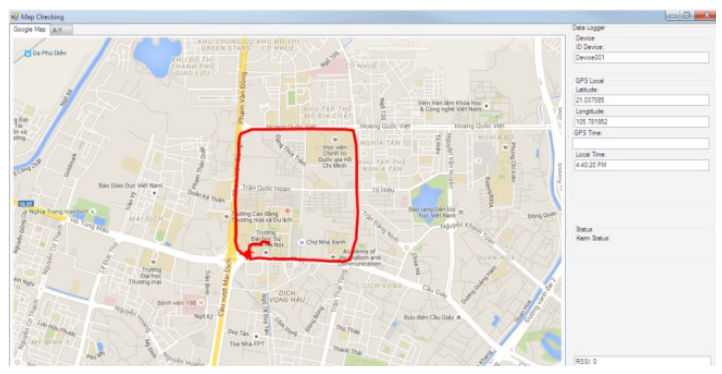

Figure 16. An example for tracking the signs of one vehicle

offline Google map, which helps the users determine the direction of movement. The red line is the route of one target. Beside the digital map, the software is capable of tracking and displaying the position of the vessels on XY axis coordinates, and calculating the distance from the vessels to the Continent. In case of danger, the control station in the Continent will receive the urgent message (SOS) and determine exactly the coordinates of the vessels at that time. After that, they will cooperate with other systems to rescue quickly and sensibly.

\section{CONCLUSION}

In this paper, the status data transmitting system suitable for vessel monitoring has been presented. The proposed frequency synthesizer can flexibly change the frequency in a wide range (from $600 \mathrm{MHz}$ to $4.2 \mathrm{GHz}$ ) and the output power (from $-6 \mathrm{dBm}$ to $-3 \mathrm{dBm}$ ). By using the phase-locked loop, the synthesizer's stability over 


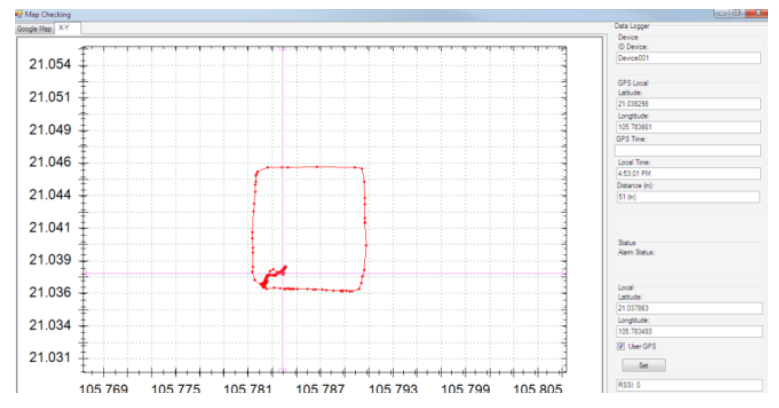

Figure 17. Displaying in XY axis coordinates

temperature and tolerance are comparable to TCXO that is about $+/-3 \mathrm{ppm}$. Moreover, phase noise performance of the synthesizer is less than $-90 \mathrm{dBc} / \mathrm{Hz}$ at $1 \mathrm{KHz}$ and $-100 \mathrm{dBc} / \mathrm{Hz}$ at $100 \mathrm{KHz}$. The impedance bandwidth of the horn antenna can be controlled by using the beveling technique. The status data module packs information of the identification, longitude, latitude, and state of the vessel into data frames. FSK/MSK/GMSK schemes were used to modulate the data. The power amplifier provides $130 \mathrm{~W}$ output power at $\mathrm{S}$ band. The fundamental characteristics of the implemented transmitter have been measured and vertified. By changing the parameters automatically, the status data transmitting system is able to communicate with both satellites and stations, solving the communication distance issue.

\section{ACKNOWLEDGEMENT}

This research is granted by Vietnam Space Science and Technology Program through the national projects titled "Research, Design, Integrate, Launch and Operate a Nano Satellite - VT-CN.02/17-20".

\section{REFERENCES}

[1] Shwu-Jing Chang, "Vessel Identification and Monitoring Systems for Maritime Security," IEEE 37th Annual International Carnahan Conference on Security Technology, pp. 66-70, October 2003.

[2] "Technical characteristic for an automatic identification system using time division multiple access in the VHF maritime mobile band," recommendation ITU-R M.1371-5.

[3] "Satellite detection of automatic identification system messages," Report ITU-R M.2084.

[4] "Satellite AIS," An exactEarth Technical White Paper, April 2015.

[5] Nguyen Dinh The Anh, Le Xuan Huy, Vu Tuan Anh and Bach Gia Duong, "Research, Design and Fabrication of a Data Transceiver Module for Vessel Monitoring Systems," The 2016 International Conference on Advanced Technologies for Communications (ATC16), pp. 524-529, October 2016.

[6] The Anh Nguyen Dinh, Giang Bach Hoang, Tuan Anh Vu and Duong Bach Gia, "A Solution to Enhance the Efficiency of the High Power S Band LDMOS Amplifier for Microwave Power Transmission and Wireless Communication," The Vietnam-Japan Microwave Workshop (VJMW2015), 2015.

[7] Giang Bach Hoang, The Anh Nguyen Dinh, Tuan Anh Vu, Duong Bach Gia "Research, Design and Fabrication of a $2.4 \mathrm{GHz} 130 \mathrm{~W}$ Power Amplifier Module for Free-Space Energy-Transmission Systems", The 5th International Conference on Integrated Circuits, design, and Verification (ICDV 2014), pp. 164-169, November 2014.

[8] Zhu Chunhua, Yang Jing, Wang Shuili, ”Key Index Analysis of PLL Frequency Synthesizer with High Resolution", Video engineering, vol. 37, pp. 98-100, November 2013.

[9] Song Qingping, Qi Jianzhong, ”ADF4350-Based Frequency Modulation Transmitter Design," IEEE Conference Publications, pp. 1-3, November 2014.

[10] Akihiro Kajiwara, Masao Nakagawa, ”A New PLL Frequency Synthesizer with High Switching Speed," IEEE Transl. J. Vehicular Technology, vol. 41, pp. 407-413, November 1992.

[11] Muhammad Kashif, Zahid Yaqoob Malik, Mubashar Yasin, Muhammad Imran Nawaz," K-Band PLL Based Frequyency Synthesizer, Proceedings of 6th International Bhurban Conference on Applied Sciences and Technology, vol. 2, pp. 136-139, 2009.

[12] Analog Devices, "ADF4350 Datasheet, 2017.

[13] STMicroelectronics, "STM32F103xx Datasheet, 2007.

[14] Cao hui, Qu Yu, "A miniaturized frequency synthesizer system design based on ADF4350," The 2016 IEEE 
International Conference on Signal Processing, Communications and Computing (ICSPCC), 5-8 August 2016.

[15] Hui Xu, Liang Peng, ’DDesign of Ultra-broadband microwave sources based on ADF4350," The 2010 2nd International Conference on Advanced Computer Control (ICACC), 27-29 March 2010.

[16] Song Qingping, Qi Jianzhong, "ADF4350-based Frequency Modulation transmitter design,” The 2014 International Conference on Cyberspace Technology (CCT 2014), 8-10 November 2014.

[17] Lin Wang, Yuanwang Yang, Jiangyue Cai and Gang Liu, ”A wide frequency coverage synthesizer with high performance for 3MHz-5GHz transceiver," The 2013 International Conference on Information Science and Technology (ICIST), 23-25 March 2013.

\section{BIOGRAPHIES OF AUTHORS}

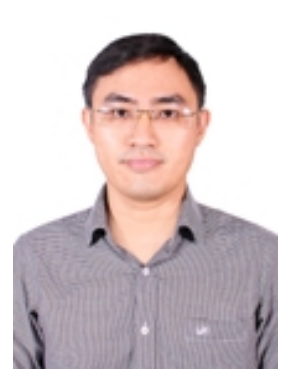

The Anh Nguyen Dinh received the B.S Degree and M.Sc Degree in Electronics and Telecommunications Technology from University of Engineering and Technology, Vietnam National University in 2009 and 2011, respectively. From 2012 to 2015, he was a researcher in Communications and Television Development., JSC. Since 2016, he has been a researcher in Vietnam National Space Center, Vietnam Academy of Science and Technology. Now, he is a Ph.D student in VNU University of Engineering and Technology. His researches are in fields of microwave engineering, communications in satellites, ground station and radio systems.

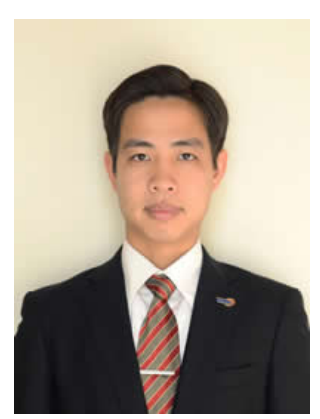

Huy Le Xuan received Ph.D. Degree in Mechanical and Aerospace Engineering from Tokyo Institute of Technology in 2014. He has been working for VNSC since 2011 at Space Systems Design Department. His research interests are small Earth observation satellites development, system engineering, satellite attitude determination and control, optimal estimation of dynamics systems. Currently, he is technical and process monitoring for both NanoDragon (4kg) and MicroDragon $(50 \mathrm{~kg})$ satellite projects in VNSC. He is also the head of Space Systems Design Department in VNSC. Involved projects: PicoDragon - $1 \mathrm{~kg}$ satellite, Tsubame - 50kg satellite of Tokyo Institute of Technology

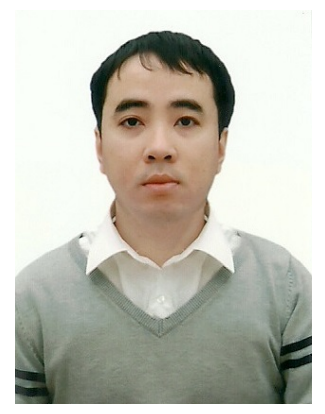

Tuan Anh Vu received the B.S Degree and M.Sc Degree in Electronics and Telecommunications Technology from University of Engineering and Technology, Vietnam National University in 2006 and 2009, respectively. In 2013, he received Ph.D Degree in the field of analog/mixed-signal RF nano electronics from University of Oslo, Norway. Since 2014, he has been a lecturer at Faculty of Electronics and Telecommunications, VNU University of Engineering and Technology. Dr. Tuan Anh Vu was with Department of Semiconductor Electronics and Integration Science, Hiroshima University as a postdoctoral researcher for one year. He is now doing postdoc at Department of Electrical and Computer Engineering, University of California, Davis. His research interests are analog RF integrated circuit designs, energy harvesting, microwave engineering, etc.

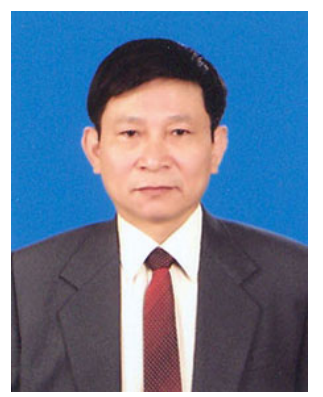

Duong Bach Gia was born in Ha Dong Dist, Ha Noi Province, Viet Nam in 1950. He received the B.S degree in radio physics in 1972 and the Ph.D degree in wireless physics from University of HaNoi in 1988. From 1988 to 1990, he was a researcher assisstant in Leningrad University, Russia. From 1991 to 2005, he was a researcher in academy of air force. He has been a lecturer and head of electronics and telecommunications center, University of Engineering and Technology, Vietnam National University since 2006. He was promoted to Associate Professor in 2009 and to Professor in 2016. His research forcuses on RF analog singal processing, RF chip design, radar engineering and technology, automatic control. Email: duongbg@vnu.edu.vn 